\title{
Multiple-imputation-particle-filtering for Uncertainty Characterization in Battery State-of-Charge Estimation Problems with Missing Measurement Data: Performance Analysis and Impact on Prognostic Algorithms
}

\author{
David E. Acuña ${ }^{1}$, Marcos E. Orchard ${ }^{1}$, Jorge F. Silva ${ }^{1}$, and Aramis Pérez ${ }^{1}$ \\ ${ }^{1}$ Universidad de Chile, Department of Electrical Engineering. Av. Tupper 2007, Santiago, Chile \\ davacuna@ing.uchile.cl \\ morchard@ing.uchile.cl \\ josilva@ing.uchile.cl \\ aramis.perez@ing.uchile.cl
}

\begin{abstract}
The implementation of particle-filtering-based algorithms for state estimation purposes often has to deal with the problem of missing observations. An efficient design requires an appropriate methodology for real-time uncertainty characterization within the estimation process, incorporating knowledge from other available sources of information. This article analyzes this problem and presents preliminary results for a multiple imputation strategy that improves the performance of particle-filtering-based state-of-charge (SOC) estimators for lithium-ion (Li-Ion) battery cells. The proposed uncertainty characterization scheme is tested, and validated, in a case study where the state-space model requires both voltage and discharge current measurements to estimate the SOC. A sudden disconnection of the battery voltage sensor is assumed to cause significant loss of data. Results show that the multipleimputation particle filter allows reasonable characterization of uncertainty bounds for state estimates, even when the voltage sensor disconnection continues. Furthermore, if voltage measurements are once more available, the uncertainty bounds adjust to levels that are comparable to the case where data were not lost. As state estimates are used as initial conditions for battery End-of-Discharge (EoD) prognosis modules, we also studied how these multiple-imputation algorithms impact on the quality of EoD estimates.
\end{abstract}

\section{INTRODUCTION}

Over the last decades, we have experienced a significant increment in the development and production of electric vehicles. The automotive industry has been allocating research ef-

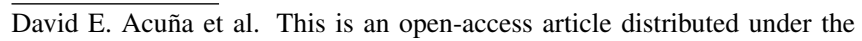
terms of the Creative Commons Attribution 3.0 United States License, which permits unrestricted use, distribution, and reproduction in any medium, provided the original author and source are credited. forts into the development of energy storage devices (ESDs) for the production of hybrid electric vehicles (HEV) or fully electric vehicles $(\mathrm{EV})$. As a result, ESDs currently play a crucial role regarding autonomy of the systems they energize. This last fact has motivated research on Li-Ion battery cells due to their advantages over other types of ESDs, being its larger charge density (by unit of mass or volume) one of the most important features to consider. From the automotive industry, the concept of "Battery Management Systems” (BMS) (Pattipati, Sankavaram, \& Pattipati, 2011) rises naturally when looking for systems capable of providing protection and optimal operating conditions for batteries, while simultaneously accounting for life predictions through the supervision of real-time acquired data. In this regard, the "State-of-Charge" (SOC) (Pattipati et al., 2011) -a measure of the remaining available energy stored-, the "State-ofHealth" (SOH) (Pattipati et al., 2011) -a measure of battery degradation-, and the associated "Remaining Useful Life" (RUL) (Orchard \& Vachtsevanos, 2009) are parameters that provide important information about the current condition of the battery. Unfortunately, due to incapability to measure them directly in an online manner, BMS systems must incorporate real-time estimation and prediction routines to carry out their objectives.

These estimation and prediction routines heavily depend on real-time measurements for their implementation and thus, these schemes are subject to information losses due to, for example, transmission problems or sensor disconnection. Completing missing values within the acquired data set is not just as simple as filling in missing data with averaged values. In this regard, many strategies can be adopted to solve the problem of sequential state estimation with incomplete data sets. Among them, single imputation methods fail due to the lack of uncertainty characterization. To solve this issue, the idea 
of multiple imputations was proposed in (Rubin, 1987). This latter method considers different values for each missing datum and combines their induced probability distributions into a single solution for parameter estimation. This technique led to the multiple imputation particle filter (Housfater, Zhang, \& Zhou, 2006), where particle-filtering methods (Andrieu, Doucet, \& Punskaya, 2001) were used, taking into account uncertainty of missing data through a multiple imputation strategy.

This work presents an improvement of the particle-filteringbased Bayesian approach adopted by (Pola et al., 2015) for real-time uncertainty characterization in SOC estimation for Li-Ion batteries, based on a multiple-imputation strategy. Two validation cases are considered for this algorithm. The first case considers a situation where 1000 sequential voltage measurements are lost, emulating the disconnection of the associated sensor during the execution of a specific discharge cycle. The second case explores the impact of losing 500 sequential voltage measurements just before the execution of a prognosis module. Obtained results show that the uncertainty associated to missing information is bounded when using the proposed method. Furthermore, obtained uncertainty bounds are smaller than those obtained when simply discarding incomplete measurements and computing the nstep-ahead prior state probability density function (PDF).

The article is structured as follows. In Section 2, a theoretical background is presented reviewing the underlying concepts of particle filters and the multiple imputation strategy. In Sections 3, a new multiple-imputation-based particle filter is presented for SOC estimation in Li-Ion battery cells when voltage and discharge current are measured. Sudden disconnections of the battery voltage sensor are simulated and uncertainty characterization is analyzed in Section 4 (both in terms of estimation and prognosis modules). Finally, conclusions and future work are presented in Section 5.

\section{THEORETICAL BACKGROUND}

Real world systems are commonly dynamic, nonlinear, and may involve a high dimensionality relationship between variables. State-space models offer a good theoretical framework for the development of diagnostic (or prognostic) modules devoted to monitor critical system components which phenomenology follow laws of fist principles. Moreover, uncertainty due to the lack of knowledge about the actual system dynamics or noisy measurements (or sensor nonlinearities) can be incorporated into the state-space form with ease. This allows to adopt Bayesian filtering approaches, where the main objective is to estimate the underlying probability distribution to perform statistical inference. Since analytical solutions may be found under certain conditions, the real problem to be addressed is that of evaluating complex integrals where numerical methods tend to breakdown, even more when high dimensional systems are involved. An alternative to address this problem is the use of particle filters, which is presented in the following section. Later, an introduction to multiple imputation for dealing with missing data and the way multiple imputation particle filter is presented.

\subsection{Particle Filters}

Due to the employment of digital computers for signal processing, it is of interest to develop a Bayesian processor where measurements arrive sequentially in time. The process of recursively estimating the evolving posterior distribution is the so called optimal filtering problem. A mathematical framework is provided below for solving this problem using particle filters.

Let $X=\left\{X_{t}, t \in \mathbb{N}\right\}$ be a first order Markov process denoting a $n_{x}$-dimensional system state vector with initial distribution $p\left(x_{0}\right)$ and transition probability $p\left(x_{t} \mid x_{t-1}\right)$. Also, let $Y=\left\{Y_{t}, t \in \mathbb{N} \backslash\{0\}\right\}$ denote $n_{y}$-dimensional conditionally independent noisy observations. The whole system is represented in state-space form as

$$
\begin{aligned}
x_{t} & =f\left(x_{t-1}, \omega_{t-1}\right) \\
y_{t} & =g\left(x_{t}, v_{t}\right)
\end{aligned}
$$

where $\omega_{t}$ and $v_{t}$ denote independent random variables whose distributions are not necessarily Gaussian. Since it is difficult to compute the filtering posterior distribution $p\left(x_{t} \mid y_{1: t}\right)$ directly, Bayesian estimators are constructed from Bayes' rule.

Under Markovian assumptions, the filtering posterior distribution can be decomposed into

$$
p\left(x_{t} \mid y_{1: t}\right)=\frac{p\left(y_{t} \mid x_{t}\right) \cdot p\left(x_{t} \mid y_{1: t-1}\right)}{p\left(y_{t} \mid y_{1: t-1}\right)}
$$

In this context, sequential Monte Carlo methods (SMC) offer an alternative to numerical integration techniques that fail in the context of real-time embedded systems due to high computation requirements. SMC methods, also called particle filters, are stochastic computational techniques designed for simulating highly complex systems in an efficient way. In Bayesian estimation, these techniques simulate probability distributions by using a collection of $N$ weighted samples or particles, $\left\{x_{t}^{(i)}, \mathcal{W}_{t}^{(i)}\right\}_{i=1}^{N}$, that yields to discrete mass probability distributions, as shown in Eq. (4).

$$
\hat{p}\left(x_{t} \mid y_{1: t}\right) \approx \sum_{i=1}^{N} \mathcal{W}_{t}^{(i)} \delta\left(x_{t}-x_{t}^{(i)}\right)
$$

The weighting process is made by applying the sequential importance resampling (SIR) algorithm, which is explained in the following subsections. 


\subsubsection{Sequential Importance Sampling}

The concept of importance sampling is used to simulate samples from a proposed distribution in order to estimate a posterior distribution. The key point for a successful sampling is to choose appropriately the importance distribution. Sampling from posterior distributions is a common task in order to get Monte Carlo (MC) estimates. However, it is not feasible most of the time since it becomes computationally intensive. For example, Eq. (5) shows the calculation of expectations.

$$
\hat{f}\left(x_{t}\right)=E_{X \mid Y}\left\{f\left(x_{t}\right)\right\}=\int_{X} f\left(x_{t}\right) p\left(x_{t} \mid y_{1: t}\right) d x_{t}
$$

Drawing $N$ independent identically distributed random samples from $p\left(x_{t} \mid y_{1: t}\right)$, the integral may be approximated by a sum of delta-Dirac functions.

$$
\begin{aligned}
\hat{f}\left(x_{t}\right) & \approx \frac{1}{N} \sum_{i=1}^{N} f\left(x_{t}\right) \delta\left(x_{t}-x_{t}^{(i)}\right) \\
& =\frac{1}{N} \sum_{i=1}^{N} f\left(x_{t}^{(i)}\right)
\end{aligned}
$$

These approximations may not hold when it is not possible to sample directly from $p\left(x_{t} \mid y_{1: t}\right)$, thus the sequential importance sampling (SIS) algorithm avoids these difficulties by drawing samples from an importance distribution approximating the targeted posterior distribution by appropriate weighting. The weights are recursively defined as

$$
w_{t}^{(i)}=w_{t-1}^{(i)} \cdot \frac{p\left(y_{t} \mid \tilde{x}_{t}^{(i)}\right) \cdot p\left(\tilde{x}_{t}^{(i)} \mid x_{t-1}^{(i)}\right)}{\pi\left(\tilde{x}_{t}^{(i)} \mid \tilde{x}_{0: t-1}^{(i)}, y_{1: t}\right)}
$$

where $\left\{\tilde{x}_{t}^{(i)}\right\}_{i=1}^{N}$ is a set of $N$ random samples drawn from the importance distribution $\pi\left(\tilde{x}_{t}^{(i)} \mid \tilde{x}_{0: t-1}^{(i)}, y_{1: t}\right)$. Also, defining normalized weights

$$
\mathcal{W}_{t}^{(i)}=\frac{w_{t}^{(i)}}{\sum_{i=1}^{N} w_{t}^{(i)}}
$$

then the posterior distribution can be approximated by the expression described in Eq. (4).

\subsubsection{Resampling}

When the updating process begins, a tendency to increase the variance of particles can be seen, setting negligible weights to some of them. These particles become useless as they track low probability paths of the state vector. In order to solve this problem, a resampling step is incorporated, which leads to the SIR algorithm.

An analytical expression for measuring how degenerated are the particles is given by the effective particle sample size shown in Eq. (10).

$$
N_{e f f}(t)=\frac{N}{1+\operatorname{Var}_{p\left(\cdot \mid y_{1: t}\right)}\left(w\left(x_{t}\right)\right)}
$$

As it is not possible to calculate $N_{e f f}$, an estimate is given by

$$
\hat{N}_{e f f}(t)=\frac{1}{\sum_{i=1}^{N}\left(\mathcal{W}_{t}^{(i)}\right)^{2}}
$$

In other words, the resampling step consist of removing small weighted particles while retaining and replicating those of large weights. Thus, whenever $\hat{N}_{\text {eff }} \leq N_{\text {thres }}$, with $N_{\text {thres }}$ a fixed threshold, the depletion of the particles is imminent and resampling must be applied.

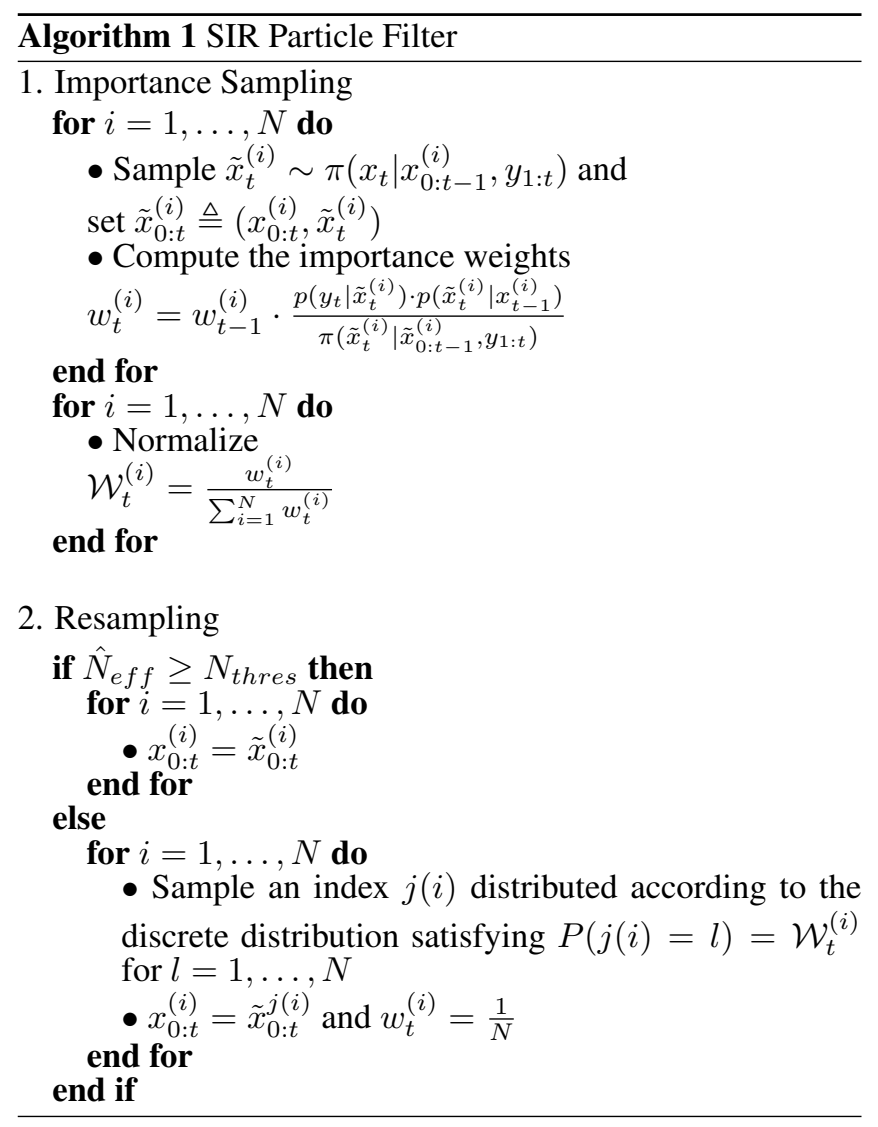

In general, the SIR particle filter is divided into two steps. Firstly, a prediction is done using the state transition model to generate the prior distribution $p\left(x_{k} \mid x_{k-1}\right)$. Then an update step is done to modify the particle weights through the likelihood $p\left(y_{k} \mid x_{k}\right)$. If the resulting particles are degenerated, a resampling step is added, as it was shown previously.

\subsection{Multiple imputations}

Missing data is a problem that may be treated mainly from two perspectives. On the one hand, single imputation techniques fill the incomplete data set imputing single values at 
each missing datum. The advantage of this perspective is that it allows standard complete data methods to be used. However, these techniques fail due to the lack of uncertainty characterization of both, the sampling variability and the uncertainty associated with the imputation model. On the other hand, the idea of multiple imputations retains the advantages of single imputation techniques and also accounts for the uncertainty of the missing mechanism. Multiple imputations (Rubin, 1987) consist of creating multiple complete data sets imputing $m$ values for each missing datum so that sampling variability around the actual values is incorporated for performing valid inferences. Nevertheless, multiple imputations has disadvantages like the need of drawing more imputations and larger memory space for storing and processing multipleimputed data sets.

An important issue is the task of choosing the right number of imputations (Graham, Olchowski, \& Gilreath, 2007). Obviously, the computational cost is higher as the number of imputations increases. In this regard, (Rubin, 1987, p. 114) shows that an approximation of efficiency for an estimate is given by

$$
\left(1+\frac{\gamma}{m}\right)^{-1 / 2}
$$

in units of standard errors, where $m$ is the number of imputations and $\gamma$ is the fraction of missing information in the estimation. Consequently, excellent results may be obtained using only few imputations $(m=3,4,5)$.

\subsection{Multiple Imputation Particle Filter}

Originally introduced by (Housfater et al., 2006), the Multiple Imputation Particle Filter extends the PF algorithm by incorporating a multiple imputation (MI) procedure for cases where measurement data is not available, so that the algorithm can include the corresponding uncertainty into the estimation process. The main statistical assumption in this approach is that the missing mechanism is Missing at Random (MAR), thus, it does not depend on the missing measures given the observed ones.

For readability, a change in notation is necessary. As it was stated in (Housfater et al., 2006), lets denote now the measurements as a partitioned vector $U_{t}=\left(Z_{t}, Y_{t}\right)$, where $Z_{t}$ corresponds to the missing part and $Y_{t}$ is from now on the observed part. Then, the MI PF algorithm performs the same as the SIR PF except that there are missing measures. In this case, a MI strategy is adopted.

An imputation model expressed as a probability distribution $\phi$ is required for drawing $m$ samples -imputations-, that is

$$
z_{t}^{j} \sim \phi\left(z_{t} \mid y_{1: t}\right)
$$

where $j=\{1, \ldots, m\}$ denotes the imputation index. Similarly to importance sampling, each imputation is associated with a weight $p_{t}^{j}$ holding the condition $\sum_{j=1}^{m} p_{t}^{j}=1$. Ac- cording to (Liu, Kong, \& Wong, 1994), the filtering posterior distribution may be expressed as

$$
p\left(x_{t} \mid y_{1: t}\right)=\int p\left(x_{t} \mid u_{1: t-1}, y_{t}\right) p\left(z_{t} \mid y_{1: t}\right) d z_{t} .
$$

By performing a Monte Carlo approximation yields

$$
p\left(x_{t} \mid y_{1: t}\right) \simeq \sum_{j=1}^{m} p_{t}^{j} p\left(x_{t} \mid u_{1: t-1}, u_{t}^{j}\right)
$$

where $u_{t}^{j}=\left(z_{t}^{j}, y_{t}\right)$ are complete data sets formed from imputed values. Additionally, by applying particle filtering to each of these data sets yields

$$
p\left(x_{t} \mid u_{1: t-1}, u_{t}^{j}\right) \approx \sum_{i=1}^{N} w_{t}^{(i, j)} \delta\left(x_{t}-x_{t}^{(i, j)}\right),
$$

where the indexes $i$ and $j$ indicate the particle and the imputation, respectively. Thus, an approximation of the desired posterior distribution is

$$
p\left(x_{t} \mid y_{1: t}\right) \approx \sum_{j=1}^{m} \sum_{i=1}^{N} p_{t}^{j} w_{t}^{(i, j)} \delta\left(x_{t}-x_{t}^{(i, j)}\right) .
$$

\section{MULTIPLE-IMPUTATION-BASED UNCERTAINTY Characterization for SOC ESTimation}

The SOC is conceived as a quantification of the available energy stored regarding the actual rated capacity, but as a percentage. It conforms an important feature to address for systems' autonomy when they are energized by ESDs, either as main sources or as a backup. As it is not possible to directly measure the SOC, estimation and prognosis algorithms must be addressed for getting valid predictions from usually noisy measurements like current, voltage and temperature, while carrying out a proper management of the system. Actually, knowledge about it is essential for control of autonomous systems where the End-of-Discharge (EoD) time plays a key role.

According to (Pola et al., 2015), a wide variety of methods have been proposed in the literature for modeling batteries in offline applications; e.g., electrochemical models. Other methods, more suitable for online implementations, are based on open-circuit voltage (OCV) representations. These methods relate directly the SOC and measured voltage but requires large resting periods for batteries, being inefficient for online estimation. The "Electrochemical Impedance Spectroscopy" (EIS) method requires costly equipment, being infeasible for practical applications. In this regard, research efforts have focused on developing estimation and prognosis algorithms based on phenomenological relations through fuzzy logic, neural networks and Bayesian frameworks (Orchard, Cerda, Olivares, \& Silva, 2012), among others. The main 
problem in all these cases is that these approaches assume complete data sets for state/parameter estimation purposes.

\subsection{State-Space Model for Lithium-Ion Batteries}

One of the main advantages of adopting a particle-filtering approach for estimation under noisy measurement data is that prior knowledge about the systems dynamics can be directly incorporated into the model as well as its associated uncertainties. Also, it is possible to capture critical physical phenomenology directly into the state-space form, relating it to an observation model which enables the convergence to the true estimates through the likelihood of sequential measurement data.

Proposed by (Pola, 2014), the state-space model for lithiumion battery cells used is the following.

\section{State transition model}

$$
\begin{aligned}
& x_{1}(t+1)=x_{1}(t)+\omega_{1}(t) \\
& x_{2}(t+1)=x_{2}(t)-\frac{v(t) \cdot i(t) \cdot \Delta t}{E_{\text {crit }}}+\omega_{2}(t)
\end{aligned}
$$

\section{Measurement equation}

$$
\begin{aligned}
v(t)= & v_{L}+\left(v_{0}-v_{L}\right) \cdot e^{\gamma \cdot\left(x_{2}(t)-1\right)}+\alpha \cdot v_{L} \cdot \ldots \\
& \ldots\left(x_{2}(t)-1\right)+(1-\alpha) \cdot v_{L} \cdot\left(e^{-\beta}-\ldots\right. \\
& \left.\ldots e^{-\beta \cdot \sqrt{x_{2}(t)}}\right)-i(t) \cdot x_{1}(t)+\eta(t)
\end{aligned}
$$

where $\omega_{1}(t) \sim \mathcal{N}\left(0, \sigma_{1}\right)$ and $\omega_{2}(t) \sim \mathcal{N}\left(0, \sigma_{2}\right)$ correspond to additive white Gaussian noise and $\eta(t) \sim \mathcal{N}\left(0, \sigma_{o b s}\right)$ is also a normal distributed random variable accounting for measurement uncertainties. The sample time $\Delta t[\mathrm{sec}]$ and the current $i(t)[A]$ are considered input variables whereas the battery voltage $v(t)[V]$ is considered the system's output. The state variables $x_{1}(t)$ and $x_{2}(t)$ are chosen strategically under physical meaning as the internal resistance and the SOC, respectively. Finally, as the SOC is expressed as a percentage of energy, $E_{\text {crit }}$ represents a normalizing constant whose units are $[V A s e c]$. All other model parameters are assumed to be known constants within each battery discharge cycle. Their values are obtained by following the procedure described in (Pola, 2014) and (Pola et al., 2015), and applying it to data that should be obtained from a complete discharge cycle at constant (nominal) discharge current.

\subsection{Implementation of a Multiple Imputation Strategy}

(Pola et al., 2015) proposed a detailed procedure for estimation and prognosis for the SOC. However, what happens when sudden disconnections (or data losses) affect sensor performance? Perhaps, SOC estimates may be eventually biased, affecting deeply the whole estimation stage and providing invalid information, and the system's autonomy would no longer be guaranteed. In this regard, a new approach from the Multiple Imputation Theory is proposed for uncertainty characterization in particle-filtering-based SOC estimators where voltage measurements are missing during extended periods of time (while discharge current measurements are always available). Future work will focus on the case when battery discharge current measurements are lost instead.

The Multiple Imputation Particle Filter uses voltage imputations in a different manner, depending on which stage of the filtering procedure is currently being applied. During the prediction stage, and if past voltage measurements are missing, the multiple-imputation algorithm suggests to draw voltage values from a proposal distribution $\phi$. Each one of these imputations will define a different prior distribution for the next time instant, since $x_{2}(t+1)$ depends on $v(t)$ in Eq. (19). However, as the state transition equations place particles in different positions of the state-space, by applying Rubin's rule of multiple imputation theory it yields, as a consequence, an increase of the particle population. This consequence is not directly an implication of Rubin's rule, but of the dependency of the prior distribution on the measurement $v(t)$.

Rubin's rule suggests that if a conditioning variable of a distribution is missing (its value is unknown), then this distribution can be approximated by an appropriate weighted sum of the distribution conditioned in several possible values. These last are called imputations (Rubin, 1987),

Assuming that the prior distribution is known and the actual voltage value is unknown, then voltage imputations may also be considered for the update stage of the particle-filtering algorithm. Furthermore, in that case the resulting particles (which represent the posterior distribution) will keep the same location within the state-space. Thus, the number of particles is not increased since Rubin's rule (Rubin, 1987) is applied.

As multiple-imputed data generate an increase of the number of particles during the prediction stage, a reduction stage has to be incorporated into the algorithm to keep a fixed number of particles throughout time; avoiding a progressive increase of the particle population. This way, the SIR PF will work as it was originally designed, especially after voltage measurements are available once more again.

In particular, the problem of reducing the number of particles from $N \cdot m$ to $N$-where $N$ is the size of the original particle population and $m$ is the number of imputations- could be achieved by resampling. However, this kind of technique fails because of the algorithm tendency to retain high probability particles only, thus discarding the uncertainty characterization provided by the MI strategy. Therefore, a suboptimal solution is proposed. The main focus consists on preserving the probability distribution described by $N \cdot m$ particles using only $N$ of them. Thus, as an attempt to solve this problem, the particles are arranged as a function of the SOC 
$\left(\left\{x_{k}^{(l)}, \mathcal{W}_{k}^{(l)}\right\}_{l=1}^{N \cdot m}\right)$ and clustered into groups of $m$ particles, noting that the SOC corresponds to a state and its dynamic is described in Eq. (19). One particle is obtained from each group by a weighted sum and its probability is assumed to be the sum of probabilities of each particle in the group. Therefore, the $N$ new particles are generated as

$$
\begin{aligned}
\overline{\mathcal{W}}_{k}^{(i)} & =\sum_{l=m \cdot(i-1)+1}^{m \cdot i} \mathcal{W}_{k}^{(l)} \\
\bar{x}_{k}^{(i)} & =\frac{1}{\overline{\mathcal{W}}_{k}^{(i)}} \cdot \sum_{l=m \cdot(i-1)+1}^{m \cdot i} \mathcal{W}_{k}^{(l)} x_{k}^{(l)}
\end{aligned}
$$

$\forall i \in\{1, \ldots, N\}$. The biggest assumption adopted for the reduction stage was that the internal impedance remains constant at least when the battery's SOC is over $20 \%$, which in practice makes it almost independent of the SOC. Of course, other factors also affect the value of the internal impedance, for example the battery temperature. In fact, that is the main reason why this parameter has to be estimated from voltage and discharge current measurements. The impact of these factors will not be considered in this particular version of the algorithm, but they will be included as part of future research work. Even though the dynamics of the internal impedance are affected by several variables (including SOC), all the results presented in this article are based on the state transition model described by Eqs. (18)-(19).

The proposed MI PF implementation solely solves the problem of missing voltage observations, whereas the discharge current is assumed in the model as an input variable that is known at each time instant. The imputation model adopted is defined through the probability distribution induced by Eq. (20), providing prior knowledge on the voltage variability that is conditional to the currently available state estimate. Thus, in the case of particle-filtering-based estimators, when substituting each particle from the prior distribution in Eq. (20), a different imputation model for the battery voltage will be generated.

By construction, the proposed algorithm shows a strong dependency -regarding its performance- on the quality of the measurement model. However, this feature is at the same time including an important source of information which is not accounted by $n$-step prediction algorithms.

Denoting the multiple-imputed measurement data set as $\tilde{y}_{1: t}^{j}=$ $\left\{\tilde{y}_{1: t-1}, y_{t}^{j}\right\}$ where $\tilde{y}_{1: t}=\left\{\tilde{y}_{1: t-1}, y_{t}^{1}, \ldots, y_{t}^{m}\right\}$, with $j \in$ $\{1, \ldots, m\}$, the MI PF implementation is summarized in Algorithm 2 .

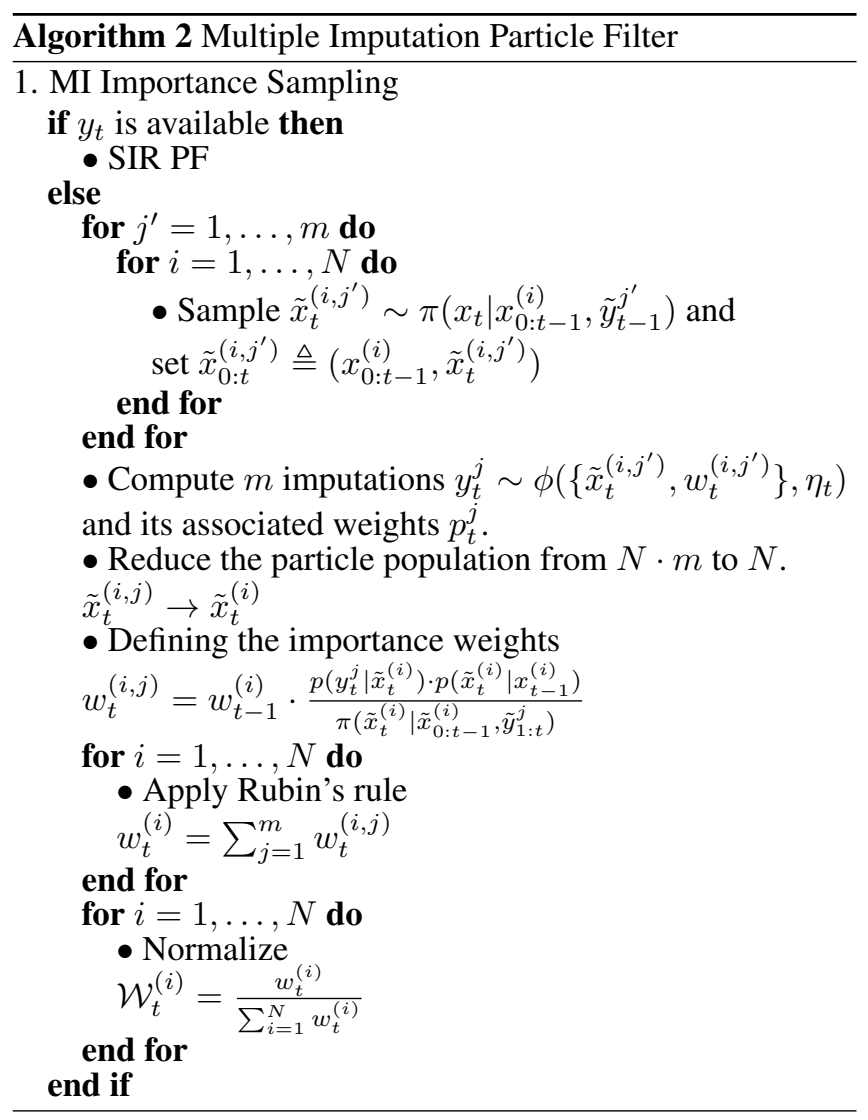

\section{EXPERIMENTAL RESUltS}

In this article, the proposed multiple-imputation algorithm is applied to the case of SOC estimation in Li-Ion battery cells. Particularly, this method is intended to improve the way SOC is monitored on a BMS. A complete discharge cycle, containing a total of 2920 samples that were obtained from an experimental setup located at the Advanced Control Systems Laboratory, University of Chile, is analyzed for purposes of algorithm test and validation.

\subsection{Impact on Estimation Stage}

To test the algorithm during the estimation stage, we consider a case where 1000 sequential voltage measurements are lost due to sensor disconnection. Estimates are obtained using 60 particles and 10 imputations. Performance is analyzed and compared considering an average of 30 realizations for three different cases: i) SIR PF with a complete data set, ii) computation of a 1000-step-ahead prior PDF in the absence of new measurements, and iii) MI PF with an incomplete data set.

The probability density that was used to draw voltage imputations corresponds to the distribution induced by Eq. (20), where particles are obtained from the prior PDF shown in Eq. (18)-(19). These particles, together with 33 realizations of the observation noise $\eta(t)$, are substituted in Eq. (20) to generate an empirical conditional distribution for the lost voltage 
measurement. The imputations are randomly drawn from the aforementioned distribution, and hence their weights are assumed to be equal.

For this case study, the conventional SIR PF is applied in all the cases as long as there are no missing measurements. The focus lays on comparing the MI strategy to a simple n-stepahead prior PDF (Orchard et al., 2012) that could be applied when voltage measurements are lost. Also, the MI strategy will be compared to the PF-based estimates that are obtained with no missing data. Both comparisons yield results for internal impedance, SOC and voltage which are exposed in Figures 1, 2 and 3, respectively. For a better analysis, the same conditions are adopted for all the cases up to the time where data starts being lost.

As it is shown in Figure 1a, the assumption of a constant value for the battery internal impedance becomes invalid as the number of lost measurements increases. In fact, MI PF estimates differ significantly from an estimate computed on the basis of a complete data set mainly because of this assumption. Also, Figure $1 \mathrm{~b}$ shows that MI PF estimates are similar, in terms of the expected value, to the ones generated using the 1000-step-ahead prior PDF. Thus, the main differences between these two methods, in terms of uncertainty characterization, are due to the hypotheses associated with the evolution of the first state. The strategy focused on the computation of a 1000-step-ahead prior PDF uses Eq. (18) for the transition of the first state, which does not represent the true dynamics of the process but a learning process called artificial evolution (Pola, 2014). If the prior PDF is characterized only using the artificial evolution concept, uncertainty will increase over time even though partial information is being collected through active sensors. Although in this case we only assumed one voltage sensor for the whole battery pack, the potential of a MI PF strategy that does not depend solely on the artificial evolution model will be more evident in a case where partial information is acquired from other sensors; which could provide, for example, information at a battery cell level.

Regardless of this fact, the main feature of the proposed MI $\mathrm{PF}$ is ensuring robust and bounded characterization of the uncertainty associated with the SOC, which is visualized in Figure 2. Figure 2a shows how MI PF uncertainty bounds overlaps the ones provided by the SIR PF, whereas in Figure $2 \mathrm{~b}$ the bounds associated to MI PF are slightly overlapped with the uncertainty characterization provided by the 1000step-ahead prior PDF. It is interesting to note the MI strategy avoids the use of a resampling stage, yielding similar results as a long term prediction. Nevertheless, when voltage measurements are not lost anymore, a bias is incorporated in both cases (MI PF and 1000-step-ahead prior PDF). This problem is generated by the assumption of constant internal impedance, which introduces a bias affecting the SOC esti-

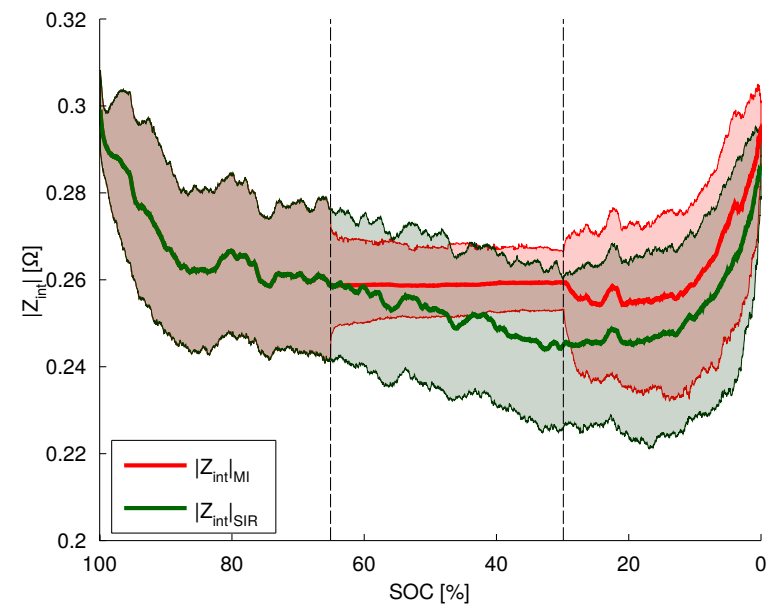

(a) MI and SIR PF

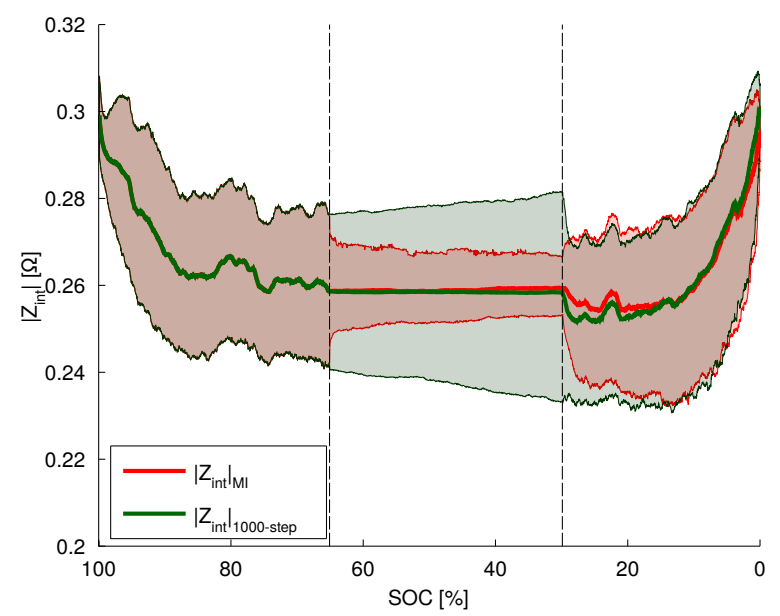

(b) MI PF and Prediction

Figure 1. Internal impedance estimation as a function of the SOC[\%] for a disconnection of 1000 sequential voltage measurements denoted in the area between the dashed vertical lines. a) Comparison between the MI PF (red line) and the SIR PF (green line) with 95\% confidence intervals. b) Comparison between the MI PF (red line) and the 1000-step-ahead prior PDF (green line) with 95\% confidence intervals. Only the SIR PF has voltage measurements available all the time whereas they remain unavailable inbetween the dashed vertical lines otherwise. The sampling rate is $1[\mathrm{~Hz}]$.

mation as an attempt to correct the first state.

The primal differences between MI PF and the n-step-ahead prior PDF method are in terms of the manner in which uncertainty is taken into account. On the one hand, the n-stepahead prior PDF (Orchard et al., 2012) method uses the state transition model to allocate the particles, replacing missing data with the expectation of the measurement equation, and neglecting the measurement noise. A regularization step is performed so that particles are equivalently weighted. These procedures are repeated along the whole prediction horizon. 


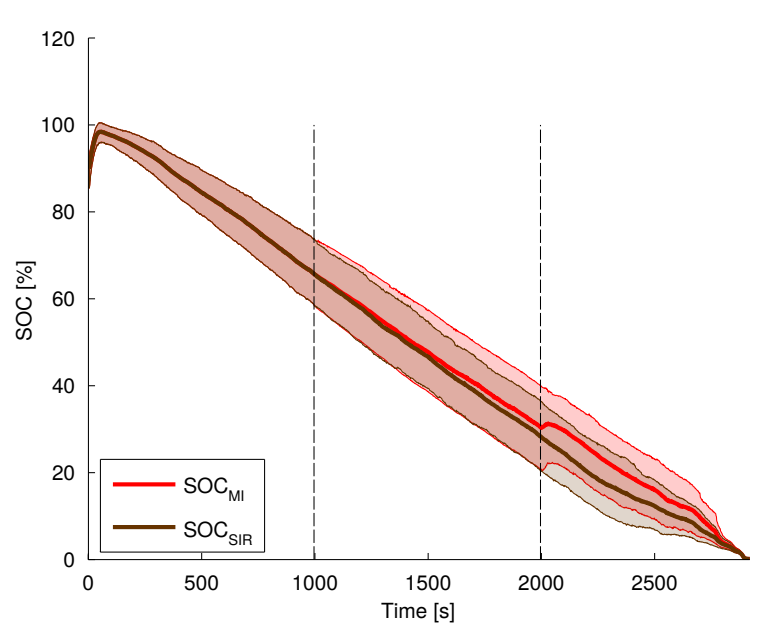

(a) MI and SIR PF

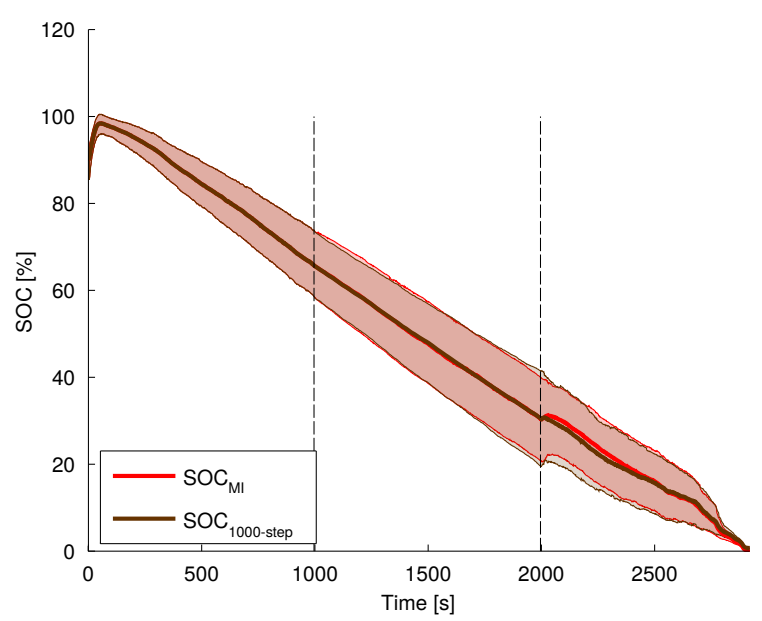

(b) MI PF and Prediction

Figure 2. SOC estimation as a function of time[s] for a disconnection of 1000 sequential voltage measurements denoted in the area between the dashed vertical lines. a) Comparison between the MI PF (red line) and the SIR PF (brown line) with $95 \%$ confidence intervals. b) Comparison between the MI PF (red line) and the 1000-step-ahead prior PDF (brown line) with $95 \%$ confidence intervals. Only the SIR PF has voltage measurements available all the time whereas they remain unavailable inbetween the dashed vertical lines otherwise. The sampling rate is $1[\mathrm{~Hz}]$.

On the other hand, the proposed implementation of MI PF, described in Section 3.2, incorporates the uncertainty that is related to the measurement system itself, simulating the convolution with typical measurement noise by sampling. The underlying importance of generating a realistic uncertainty characterization during the estimation stage is that the algorithm provides appropriate conditions for the initial conditions of the prognosis stage.

In the case of voltage estimation, results are shown in Figure 3. It can be noted in Figure 3 a that a small bias is added to the voltage distribution when using MI PF, mainly because of the bias associated with the internal impedance estimate. The use of a few imputations ( 10 in this case study) provides a reasonable characterization of the output variability by generating a robust approximation to the true statistics even when data is partially lost. The bias remains negligible considering that the total amount of lost data reaches 1000 . However, Figure $3 b$ shows that the 1000-step-ahead prior PDF shares its behavior, by describing nearly identical curves.

\subsection{Impact on Prognosis Stage}

In previous section we analyzed the effect of losing data consecutively in a window of 1000 samples during the estimation stage. We also analyzed the problems arising from this situation, yielding biased estimates and hence lack of uncertainty characterization in some cases. But what if instead of estimating after losing measurements, a prognosis routine is executed?

When information and decision making systems are involved, knowledge about future behavior of the system plays a crucial role for planning control strategies that ensure an optimal use of the available resources. In this regard, this section focuses on exposing the performance of a prognosis routine described in (Pola et al., 2015) for SOC estimation of Li-Ion batteries having lost 500 voltage measurements just before the prognosis module is executed.

For testing the algorithm during the prognosis stage, a loss of 500 sequential voltage measurements is considered just before the estimation stage is finished. The results, as in the previous section, are obtained with the use of 60 particles and 10 imputations. The performance is analyzed considering the average of 30 realizations for three different cases: i) SIR PF with a complete data set, ii) 500-step-ahead prior PDF procedure along the missing measurements, and iii) MI PF with an incomplete data set. Each realization includes in turn the mean performance of 30 realizations of prognosis in order to get results closer to the expected ones.

When the prognosis module is executed, the actual discharge current is unknown and, therefore, it must be simulated using Markov chains that help to characterize the past battery usage profile. For this reason, several realizations of the Markov Chain are required before the EoD time is adequately prognosticated. More information about this particular topic can be found in (Pola et al., 2015).

Figure 4 shows the effect of missing measurements on prognostic results in the case of the state related to the battery internal impedance. As it was mentioned before, the use of artificial evolution as a state transition model forces the expectation of the predicted distribution to remain constant, although its uncertainty steadily increases over time. Different is the case of the second state, which is associated with the 


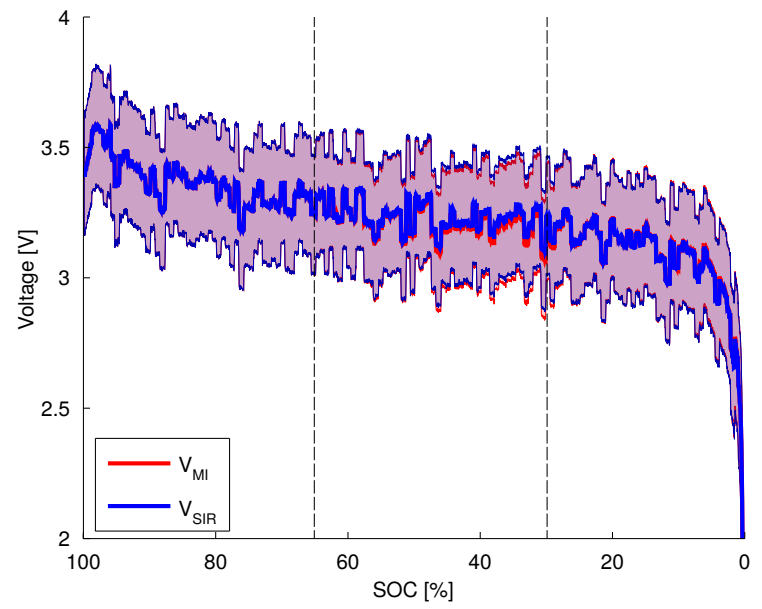

(a) MI and SIR PF

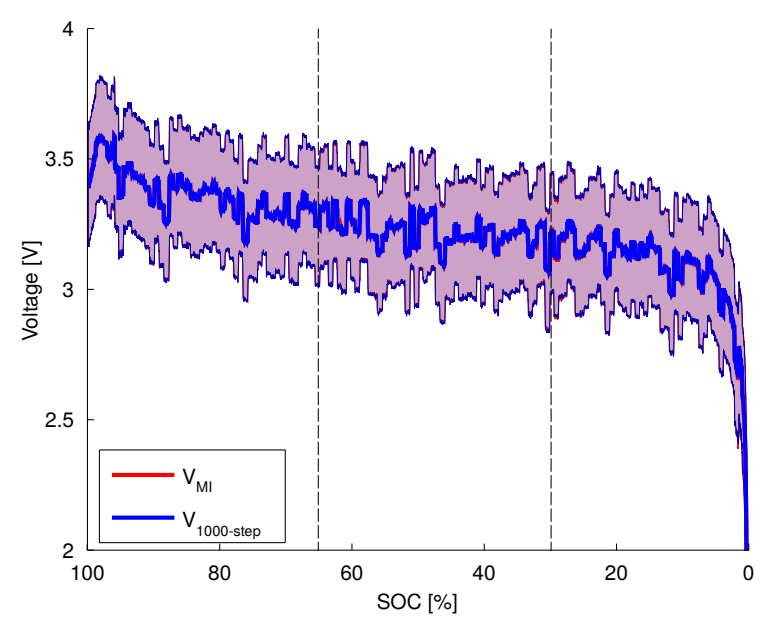

(b) MI PF and Prediction

Figure 3. Voltage estimation as a function of the SOC[\%] for a disconnection of 1000 sequential voltage measurements denoted in the area between the dashed vertical lines. a) Comparison between the MI PF (red line) to the SIR PF (blue line) with $95 \%$ confidence intervals. b) Comparison between the MI PF (red line) and the 1000-step-ahead prior PDF (blue line) with $95 \%$ confidence intervals. Only the SIR PF has voltage measurements available all the time whereas they remain unavailable inbetween the dashed vertical lines otherwise. The sampling rate is $1[\mathrm{~Hz}]$.

battery SOC; see Figure 5. Although when comparing the results of SIR PF and MI PF (see Figure 5a) in terms of the expectation of the predicted PDF these results only offer minor differences, there are slight dissimilarities in terms of the length of the confidence intervals that are built from the predicted distributions for the SOC. These slight differences in terms of the length of the confidence intervals are the consequences of having lost data before running the prognosis routine. Similar conclusions can be obtained from the analysis of the predicted battery voltage; see Figure 6 . In this regard, it

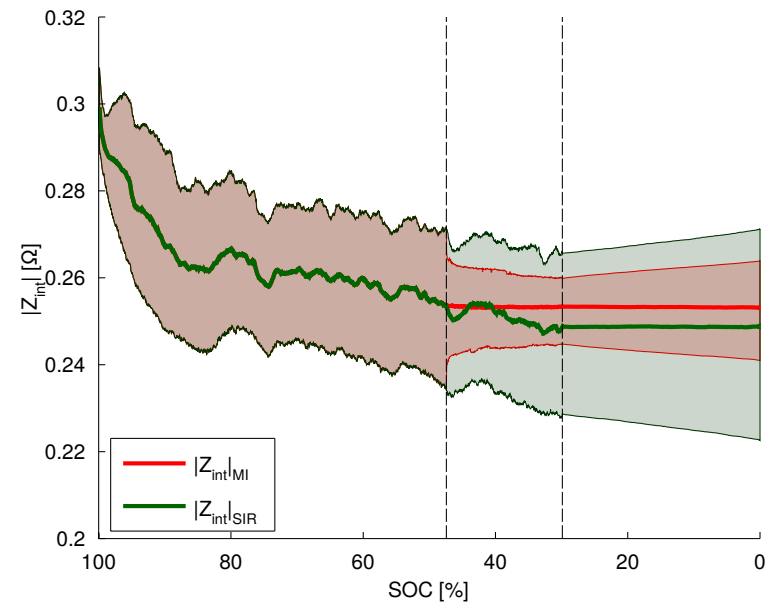

(a) MI and SIR PF

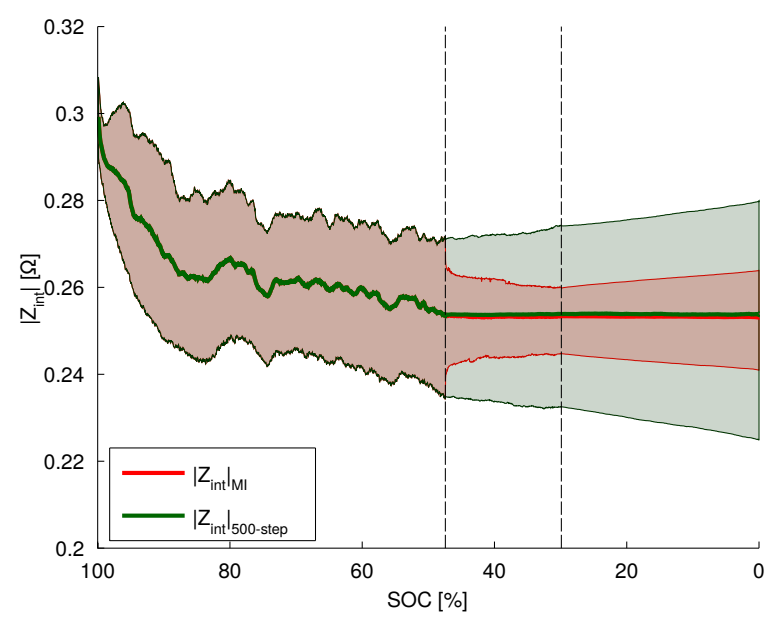

(b) MI PF and Prediction

Figure 4. Internal impedance estimation and prognosis as a function of the SOC[\%] for a disconnection of 500 sequential voltage measurements denoted in the area between the dashed vertical lines. a) Comparison between the MI PF (red line) and the SIR PF (green line) with 95\% confidence intervals. b) Comparison between the MI PF (red line) and the 500-stepahead prior PDF (green line) with 95\% confidence intervals. Only the SIR PF has voltage measurements available all the time whereas they remain unavailable inbetween the dashed vertical lines otherwise. The sampling rate is $1[\mathrm{~Hz}]$.

is important to note that the proposed method does not affect negatively the accuracy of the prediction. Moreover, in cases where the evolution of one of the states is based on the artificial evolution concept (which is helpful when learning the true value of a model parameter, but inaccurate to describe its future evolution), it helps to bound the uncertainty associated to the long-term prediction, thus improving the precision of prognostic results. 


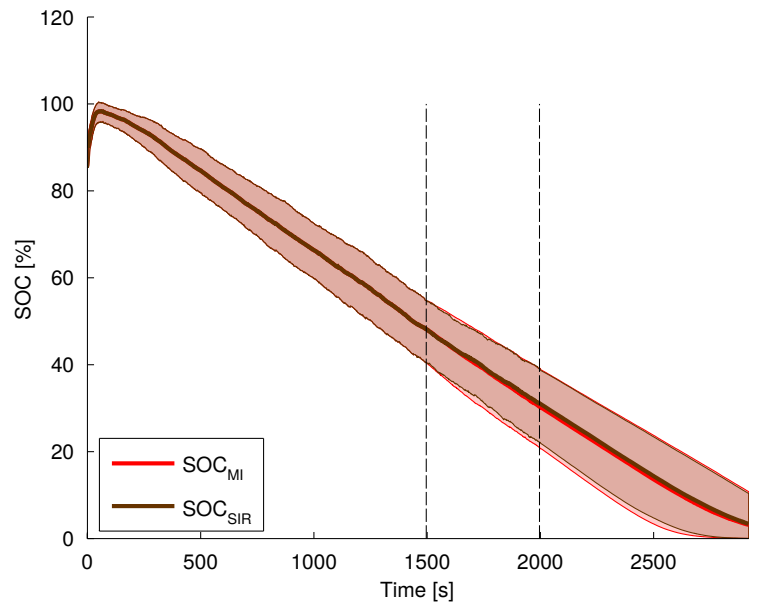

(a) MI and SIR PF

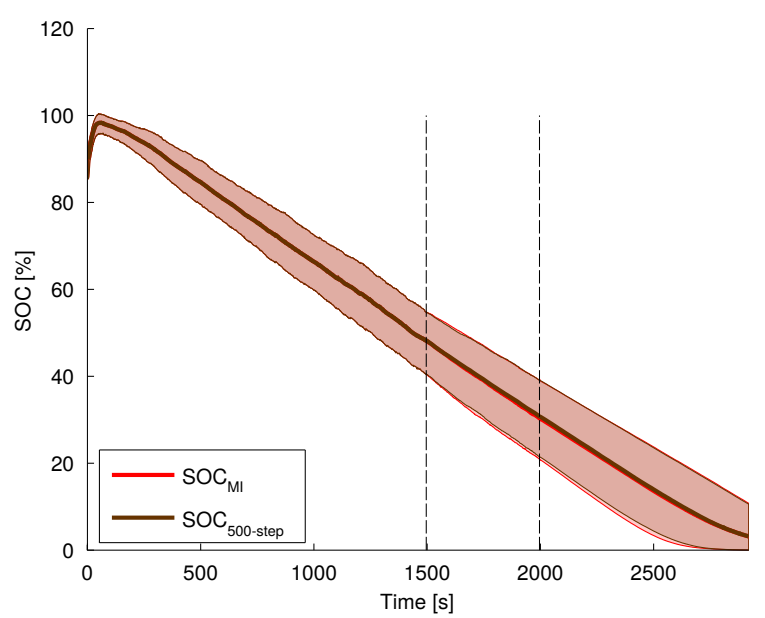

(b) MI PF and Prediction

Figure 5. SOC estimation and prognosis as a function of time[s] for a disconnection of 500 sequential voltage measurements denoted in the area between the dashed vertical lines. a) Comparison between the MI PF (red line) and the SIR PF (brown line) with 95\% confidence intervals. b) Comparison between the MI PF (red line) and the 500-step-ahead prior PDF (brown line) with 95\% confidence intervals. Only the SIR PF has voltage measurements available all the time whereas they remain unavailable inbetween the dashed vertical lines otherwise. The sampling rate is $1[\mathrm{~Hz}]$.

\section{Conclusion}

A new multiple-imputation particle-filtering based scheme for estimation with missing measurement data is proposed. In this approach, Multiple Imputation Theory is the main core for uncertainty characterization. A particular implementation for SOC estimation is presented when voltage measures are sequentially lost along a period of time. Preliminary results show the success of the methodology by incorporating uncertainty by increasing the original number of particles, but then adding a reduction stage.

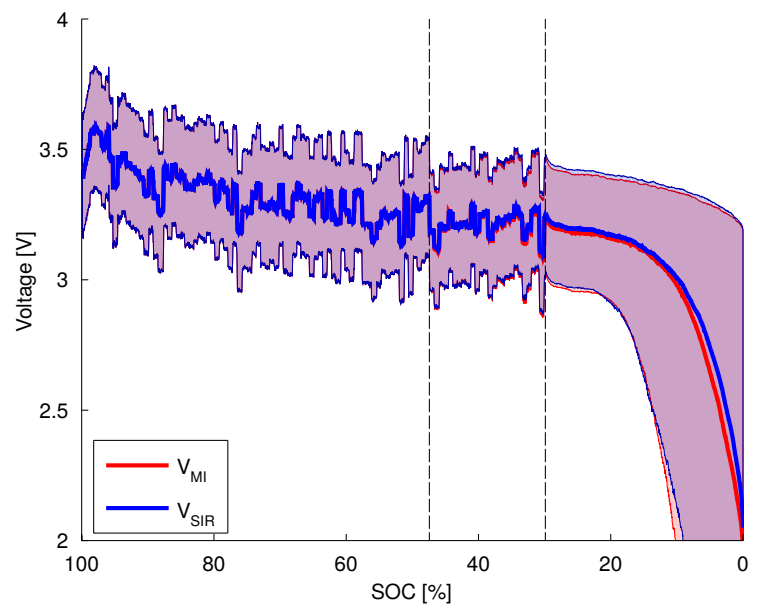

(a) MI and SIR PF

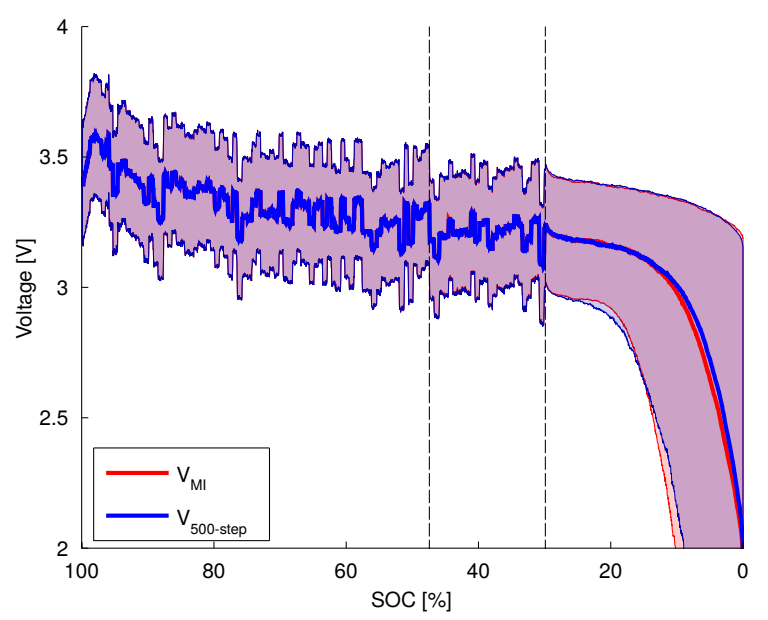

(b) MI PF and Prediction

Figure 6. Voltage estimation and prognosis as a function of the SOC[\%] for a disconnection of 500 sequential voltage measurements denoted in the area between the dashed vertical lines. a) Comparison between the MI PF (red line) to the SIR PF (blue line) with 95\% confidence intervals. b) Comparison between the MI PF (red line) and the 500-step-ahead prior PDF (blue line) with 95\% confidence intervals. Only the SIR PF has voltage measurements available all the time whereas they remain unavailable inbetween the dashed vertical lines otherwise. The sampling rate is $1[\mathrm{~Hz}]$.

The case study for testing the algorithm includes a window with missing data when the SOC is over a $20 \%$ of the battery's capacity. This allows the adoption of a simplified way for reducing particles in the algorithm based on the hypothesis that the value of the internal impedance remains constant. The MI strategy is compared to the case where the filter has access to a complete measurement data set, and also to a particle-filtering-based algorithm that computes a 1000step-ahead prior PDF when data are lost. Although MI PF estimates are similar to the ones generated using the 1000- 
step-ahead prior PDF in terms of the expected value, the main differences between these two methods are found in terms of uncertainty characterization. If the prior PDF is characterized only using the artificial evolution concept (as in the case of 1000-step-ahead prior PDF), uncertainty will increase over time even though partial information is being collected through active sensors. Although in this case we only assumed one voltage sensor for the whole battery pack, the potential of a MI PF strategy that does not depend solely on the artificial evolution model will be more evident in a case where partial information is acquired at a battery cell level. In terms of the impact of prognostic routines, the proposed method does not affect negatively the accuracy of the prediction. Moreover, in cases where the evolution of one of the states is based on the artificial evolution concept, it helps to bound the uncertainty associated to the long-term prediction, thus improving the precision of prognostic results.

As the MI has been developed for offline applications, there are several aspects that have to be considered before its implementation in online applications. Some of these aspects include improvements on the imputation model, adaptive estimation for an optimal number of particles and amount of imputations, alternative reduction methods of particle population, better ways for characterizing the internal impedance evolution in time, risk assessment, among others. Furthermore, the development of an optimal particle reduction may enable the connection of asynchronous networks, treatment for missing measurements, and prognosis, to give some examples.

\section{ACKNOWLEDGMENTS}

The authors would like to thank FONDECYT 1140774 and University of Costa Rica (Grant for Doctoral Studies) for their financial support.

\section{REFERENCES}

Acuña, D., Orchard, M., Silva, J., \& Pérez, A. (2014). Multiple-imputation-particle-filtering scheme for uncertainty characterization in battery state-of-charge estimation problems with missing measurement data. Annual Conference of the Prognostics and Health Management Society 2014, 5(037), 1-9.

Andrieu, C., Doucet, A., \& Punskaya, E. (2001). Sequential monte carlo methods in practice (A. Doucet, N. de Freitas, \& N. Gordon, Eds.). Springer-Verlag.

Candy, J. (2009). Bayesian signal processing: Classical, modern and particle filtering methods. Wiley.

Crisan, D., \& Doucet, A. (2002). A survey of convergence results on particle filtering methods for practitioners. IEEE Transactions on Signal Processing, 50(3), 736746.

Doucet, A., Godsill, S., \& Andrieu, C. (2000). On sequential monte carlo sampling methods for bayesian filtering. Statistics and Computing, 10(2), 197-208.

Graham, J., Olchowski, A., \& Gilreath, T. (2007). How many imputations are really needed? some practical clarifications of multiple imputation theory. Prevention Science, 8, 206-213.

Housfater, A., Zhang, X., \& Zhou, Y. (2006). Nonlinear fusion of multiple sensors with missing data. IEEE International Conference on Acoustics, Speech and Signal Processing, 4, 961-964.

Liu, J., Kong, A., \& Wong, W. (1994). Sequential imputations and bayesian missing data problems. Journal of the American Statistical Association, 89(425), 278-288.

Orchard, M., Cerda, M., Olivares, B., \& Silva, J. (2012). Sequential monte carlo methods for discharge time prognosis in lithium-ion batteries. International Journal of Prognostics and Health Management, 3, 1-12.

Orchard, M., \& Vachtsevanos, G. (2009). A particle-filtering approach for on-line fault diagnosis and failure prognosis. Transactions of the Institute of Measurement and Control, 31, 221-246.

Pattipati, B., Sankavaram, C., \& Pattipati, K. (2011). System identification and estimation framework for pivotal automotive battery management system characteristics. IEEE Transactions on Systems, Man, and Cybernetics, Part C: Applications and Reviews, 41(6), 869-884.

Pola, D. (2014). An improved prognosis strategy with temperature-dependent state space models for the analysis of the state-of-health and state-of-charge in lithium-ion batteries. M.Sc. Tesis. Department of Electrical Engineering, Universidad de Chile.

Pola, D., Navarrete, H., Orchard, M., Rabié, R., Cerda, M., Olivares, B., \& Silva, J. (2015). Particle-filteringbased discharge time prognosis for lithium-ion batteries with a statistical characterization of use profiles. IEEE Transactions on Reliability, 1-11.

Rubin, D. (1987). Multiple imputation for nonresponse in surveys. Wiley.

Schafer, J. (1997). Analysis of incomplete multivariate data. Chapman \& Hall/CRC.

Zhang, X., Khwaja, A., Luo, J., \& Housfater, A. (2014). Convergence analysis of multiple imputations particle filters for dealing with missing data in nonlinear problems. IEEE International Symposium on Circuits and Systems (ISCAS)(037), 2567-2570. 


\section{BIOGRAPHIES}

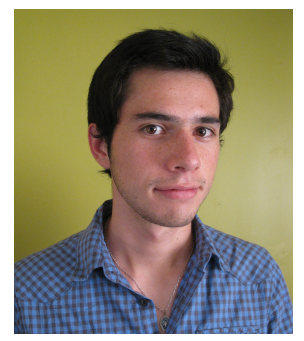

B.Sc. David E. Acuña was born in Santiago, Chile. He received his B.Sc. degree in Electrical Engineering (2014) from Universidad de Chile. He currently pursues a M.Sc. degree in Electrical Engineering at the same university and is a Research Assistant at the Lithium Innovation Center (Santiago, Chile). His research interests include signal processing, nonlinear filtering, and prognostics and health management for energy storage devices.

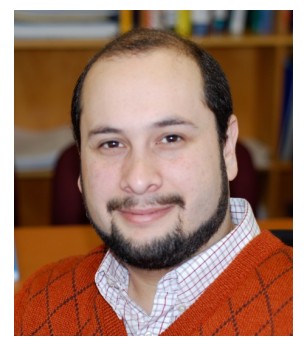

Dr. Marcos E. Orchard is Associate Professor with the Department of Electrical Engineering at Universidad de Chile and was part of the Intelligent Control Systems Laboratory at The Georgia Institute of Technology. His current research interest is the design, implementation and testing of real-time frameworks for fault diagnosis and failure prognosis, with applications to battery management systems, mining industry, and finance. His fields of expertise include statistical process monitoring, parametric/non-parametric modeling, and system identification. His research work at the Georgia Institute of Technology was the foundation of novel real-time fault diagnosis and failure prognosis approaches based on particle filtering algorithms. He received his Ph.D. and M.S. degrees from The Georgia Institute of Technology, Atlanta, GA, in 2005 and 2007, respectively. He received his B.S. degree (1999) and a Civil Industrial Engineering degree with Electrical Major (2001) from Catholic University of Chile. Dr. Orchard has published more than 50 papers in his areas of expertise.

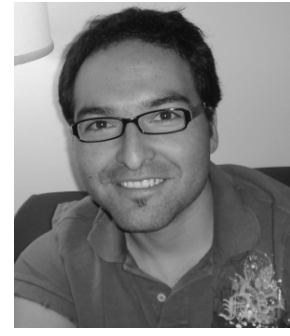

Dr. Jorge F. Silva is Assistant Professor at the Department of Electrical Engineering, University of Chile, Santiago, Chile. He received the Master of Science (2005) and Ph.D. (2008) in Electrical Engineering from the University of Southern California (USC). He is IEEE member of the Signal Processing and Information Theory Societies and he has participated as a reviewer in various IEEE journals on Signal Processing. Jorge F. Silva is recipient of the Outstanding Thesis Award 2009 for Theoretical Research of the Viterbi School of Engineering, the Viterbi Doctoral Fellowship 2007-2008 and Simon Ramo Scholarship 2007-2008 at USC. His research interests include: non-parametric learning; sparse signal representations, statistical learning; universal source coding; sequential decision and estimation; distributive learning and sensor networks.

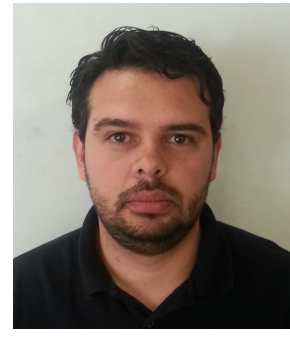

M. Sc. Aramis Pérez is a Research Assistant at the Lithium Innovation Center (Santiago, Chile) and professor at the School of Electrical Engineering at the University of Costa Rica. He received his B.Sc. degree (2002) and Licentiate degree (2005) in Electrical Engineering from the University of Costa Rica. He received his M.Sc. degree in Business Administration with a General Management Major (2008) and also he is a M.Sc. candidate in Industrial Engineering from the same university. Currently he is a doctorate student at the Department of Electrical Engineering at the University of Chile under Dr. Marcos E. Orchard supervision. His research interests include parametric/non-parametric modeling, system identification, data analysis, machine learning and manufacturing processes. 